\title{
Comparison of Electrical Breakdowns Produced by a Nanosecond High-voltage Pulse Applied to Metallic and Composite Material Electrodes
}

\author{
Abdeldjalil Reguig, Belikse Ramljak, Karl P. Chatelain, Jason S. Damazo, Eddie Kwon \\ and Deanna A. Lacoste
}

\begin{abstract}
In the present study, the effect of electrode material on the electrical breakdown, produced by a 500-ns duration highvoltage pulse in dry air at atmospheric pressure, is investigated. The configuration chosen is a pin-to-plane geometry with a gap distance of $2 \mathrm{~mm}$. Both polarities of the high-voltage pulse have been investigated for three different pin electrodes. The reference pin is a copper wire of $50 \mathrm{~mm}$ length, while the two other pins are made of highly-resistive composite material of $240 \mathrm{k} \Omega / \mathrm{m}$, with two different lengths of 50 and $500 \mathrm{~mm}$. The plane electrode is a tungsten plate of 3-cm diameter. The discharges obtained for the highly resistive wires can be categorized as resistive barrier discharges. Both electrical and optical characteristics of the discharges are presented and discussed. The current, voltage and energy deposition are first analyzed. Then, time-resolved phaselocked images of the discharges are presented showing the propagation of the discharge filaments in the gap. The experimental results demonstrate a strong influence of the electrode material on the discharge characteristics, regardless of the polarity of the applied voltage. The main finding is that, for the same applied high-voltage pulse, the use of highly resistive materials significantly reduces the energy deposition into the discharge, as well as the light emission from the discharge.
\end{abstract}

Index Terms - Resistive barrier discharges, breakdown voltage, electrical circuit, nanosecond discharges.

\section{INTRODUCTION}

$\mathrm{W}$ ITH the replacement of aluminum parts by composite materials, aircrafts face a change in the ignition threat, due to the potential charging of dielectric surfaces by static electricity [1]. The electrostatic charge accumulates through various processes including air friction during the flight, triboelectric charging as occurs during the filling of fuel tanks, or lightning events [2]. These phenomena tend to build up electrical potentials that can become high enough to cause

This paper was submitted for review on February 12, 2019. This work was supported by The Boeing Company and the King Abdullah University of Science and Technology.

Abdeldjalil Reguig is with the King Abdullah University of Science and Technology, Clean Combustion Research Center, Thuwal 23955-6900, Saudi Arabia (abdeldjalil.reguig@kaust.edu.sa).

Belikse Ramljak is with the King Abdullah University of Science and Technology, Clean Combustion Research Center, Thuwal 23955-6900, Saudi Arabia (belikse.ramljak@kaust.edu.sa). inadvertent explosion of flammable vapor spaces. According to the current regulations, like the ARP-5416 from SAE Aerospace (Aircraft Lightning Test Methods), if the energy deposition into an electrical discharge is less than $200 \mu \mathrm{J}$, the ignition hazard is below the hazardous threshold for aviation fuel tanks. This energy threshold should not be reached if the stored electrical energy on the surface is below one millijoule [3]. Other studies [4], [5] have shown that energy alone is not a sufficient quantity to determine the onset of ignition. Power density may be a more appropriate way to quantify an ignition threat.

A way to control the power density of electrostatic charges is to use the electrical properties of materials, such as their resistivity, to tune the discharge characteristics. The electrical discharges generated when using such unconventional materials as electrodes are known as resistive barrier discharges (RBDs) [6]-[12]. In 2002, Laroussi et al. [6] described the RBDs generated through a highly-resistive material (a few $\mathrm{M} \Omega / \mathrm{cm}$ ), powered by an $\mathrm{AC}$ transformer at $60 \mathrm{~Hz}$. For a planeplane electrode configuration, they showed that RBDs have the propensity to avoid arcing and to propagate in air-containing mixture with multiple filaments. Alexeff et al. [7] showed that RBDs can be powered by both AC and DC power supplies. They found that in the case of DC power supply, the plasma is self-pulsing, with a frequency that is dependent on the current. For a current intensity of $0.1 \mathrm{~A}$, in helium, the pulse frequency was about $1 \mathrm{MHz}$. They also estimated that half of the applied power was dissipated into the resistive barrier, made of a ceramic plate. In Stephan et al. [8], a glow discharge, as defined in [13], was obtained with a resistive barrier in air at atmospheric pressure, using a $3-\mathrm{cm}$ thick piece of basalt (resistivity in the range of $10^{4}$ to $10^{6} \Omega \cdot \mathrm{m}$ ), and powered by a variable DC power supply. The effect of the resistive barrier on

Karl Chatelain is with the King Abdullah University of Science and Technology, Clean Combustion Research Center, Thuwal 23955-6900, Saudi Arabia (karl.chatelain@kaust.edu.sa).

Jason Damazo is with The Boeing Company, Boeing Research and Technology, Seattle, WA, USA (jason.s.damazo@ boeing.com).

Eddie Kwon is with The Boeing Company, Boeing Research and Technology, Seattle, WA, USA (eddie.kwon@ boeing.com).

Deanna Lacoste is with the King Abdullah University of Science and Technology, Clean Combustion Research Center, Thuwal 23955-6900, Saudi Arabia (deanna.lacoste@kaust.edu.sa). 
the transverse stabilization of the glow discharge was explained by a surface-electric-field effect.

Thus, the main effects of a resistive barrier on the discharge characteristics have been identified and discussed for specific configurations. However, a quantitative understanding of RBDs is not achieved yet. The effect of various parameters such as the electrical properties of the material or the type of applied voltage on the electrical breakdown as well as on the energy deposition in the gas and in the electrodes are yet to be investigated. The objective of this study is to compare and characterize the discharges obtained with metallic electrodes and RBDs. The electrical discharges are produced in air at atmospheric pressure by a high-voltage pulse of 500-ns duration. This pulse duration has been chosen to be representative of an electrostatic discharge (single pulse with a duration between $1 \mathrm{~ns}$ and $1 \mathrm{~ms}$ ) [14], [15]. A pin-to-plane canonical configuration allows to compare the discharges obtained with a copper pin and those obtained with pins made of a highly-resistive composite wire.

\section{EXPERIMENTAL SETUP}

The experimental setup used in this study is presented in Fig. 1. Experiments are carried out in a polycarbonate chamber filled with dry synthetic air, at atmospheric pressure. All pin electrodes have a diameter of about $0.7 \mathrm{~mm}$. The plane electrode is a circular tungsten plate of $3-\mathrm{cm}$ diameter with smooth edges (3-mm radius of curvature) in order to have negligible edge effect on the electric field in the gap area. The gap distance is kept constant at $2 \mathrm{~mm}$. The high-voltage pulse of 500-ns duration is generated by an Eagle Harbor Technologies power supply (model NSP-120-20-P). The rise time of the voltage pulses is about $50 \mathrm{~ns}$. Single pulses are investigated, therefore a minimum delay of $1 \mathrm{~s}$ between two pulses is ensured. The voltage amplitude is variable by a $1 \mathrm{kV}$ step, up to $20 \mathrm{kV}$. In order to minimize the signal reflection from the load and obtain a reasonably square-shape pulse, the impedance matching is realized by adding a non-inductive $2 \mathrm{k} \Omega$ resistor (Kanthal) in parallel with the electrodes.

The three tested wires are: a) a copper wire braid of 50-mm length (referred to as $\mathrm{CuW}$ ), b) a highly-resistive wire braid of 50-mm length (HRW-50mm), and c) a highly-resistive wire braid of 500-mm length (HRW-500mm). Figure 1c shows a picture of the three tested wires. Each braid is composed of 12 wires of $0.18-\mathrm{mm}$ diameter, arranged in a circular shape. The braid itself will be referred to as "wire" in the following sections. For confidentiality reasons, the material of the highlyresistive wires cannot be disclosed.

The electrical characterization of the circuit and the discharges has been obtained by measuring the voltage and current as shown in Figs. 1a and 1b. The current probe used is a fast current transformer (Pearson, model 6585). The voltage across the inter-electrode gap, $V_{\text {gap }}$, is measured at $1 \mathrm{~mm}$ before the wire extremity. At the same time, the total voltage, $V_{t o t}$, is measured at the other wire extremity in order to evaluate the voltage drop through the tested wire. These voltage measurements are performed using two high-voltage probes (Tektronix, model P6015A). Current and voltage waveforms are recorded using an oscilloscope (Tektronix, model DPO5204B, $2 \mathrm{GHz}$ bandwidth).

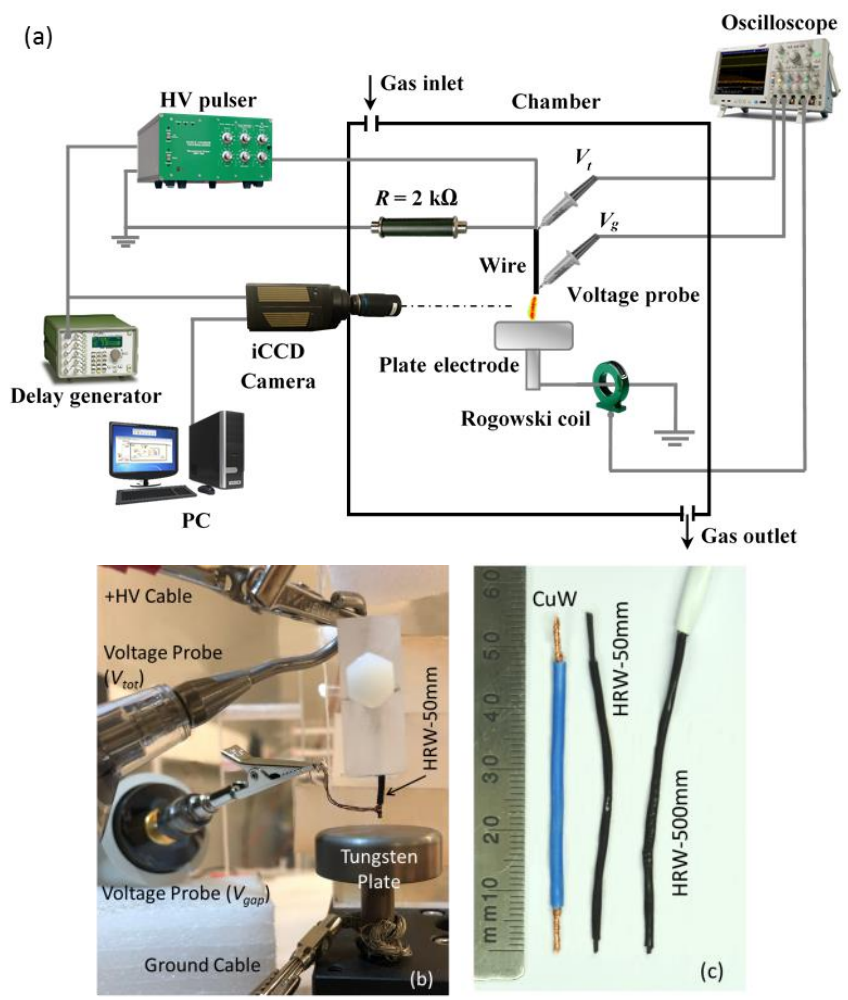

Fig. 1. a) Schematic of the experimental setup for the pin anode, b) picture of the electrode area with the HRW-50mm as anode, and c) picture of the three pin electrodes.

In addition to the electrical characterization, the temporal evolution of the discharge shape and the global light emission have been investigated. Single shot phase-locked imaging of the discharges is performed using an Intensified-CCD camera (Princeton Instruments, PIMAX), equipped with an UV lens $(105 \mathrm{~F} / 4)$. The opening gate of the camera is synchronized with the electrical system through a delay generator $(\mathrm{BNC}$, model 575) [16]. The exposure time is kept fixed at $2 \mathrm{~ns}$. For the cases with the $\mathrm{CuW}$ anode, due to the intense light emission of the discharge, neutral density filters are placed in the optical path in order to attenuate the signal below the saturation level of the camera.

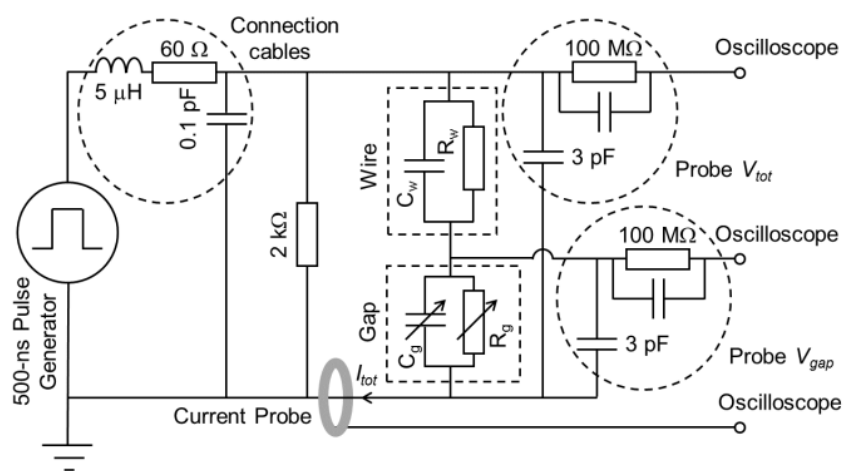

Fig. 2. Simplified equivalent electrical circuit of the experimental setup with current and voltage probes. 


\section{RESULTS AND DISCUSSION}

\section{A. Characterization of the electrical properties of the electrodes}

The equivalent capacitance and resistance of the three electrodes have been determined by combining measurements and simulations. A simplified equivalent electrical circuit of the experimental setup including the probes is depicted in Fig. 2. The values of the equivalent circuit for the connection cables have been adjusted in order to obtain the best fit between simulated and experimental current obtained for $\mathrm{CuW}$. The equivalent electrical circuit for the voltage probes are taken from the user manual.

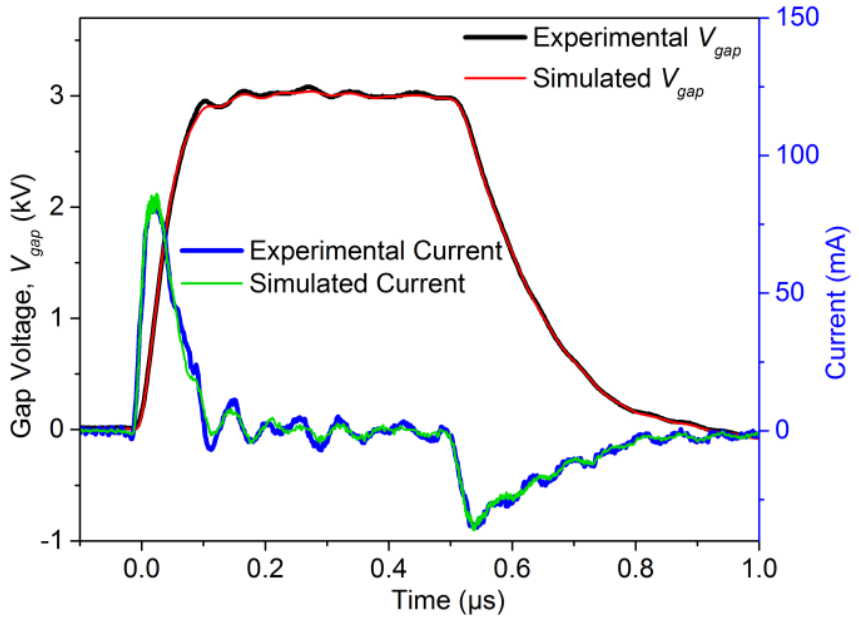

Fig. 3. Temporal evolution of the gap voltage and current, measured and calculated for the highly-resistive wire of 50-mm length.

First, the equivalent resistance of the tested wires, $R_{w}$, has been measured experimentally. For $\mathrm{CuW}$, the equivalent resistance of the $50-\mathrm{mm}$ copper braid is about $1 \mathrm{~m} \Omega$. The resistivity of the highly resistive wire has been measured at $7.3 \times 10^{-2} \Omega \cdot \mathrm{m}$. For HRW-50mm, the distributed resistance, is $\mathrm{R}_{\mathrm{w}}=12 \pm 2 \mathrm{k} \Omega$ and for HRW-500mm, $\mathrm{R}_{\mathrm{w}}=120 \pm 20 \mathrm{k} \Omega$.

When there is no discharge, the resistance of the gap, $R_{g}$, can be calculated with the Ohm's law:

$$
R_{g}=\frac{\rho_{\text {Air }} d}{\pi r^{2}}
$$

where the resistivity of the dry air, $\rho_{\text {Air, }}$ is taken equal to $2 \times 10^{16} \Omega \mathrm{m}, d$ is the gap distance, and $r$ is the channel radius, $0.7 \mathrm{~mm}$, estimated from the images of the discharges. With these numerical values, $R_{\mathrm{g}}$ is found equal to $2.5 \times 10^{19} \Omega$.

The capacitance of the gap, $\mathrm{C}_{\mathrm{g}}$, can be obtained from:

$$
I_{t o t}(t)=C_{g} \frac{d V_{g a p}(t)}{d t},
$$

where $\mathrm{I}_{\text {tot }}$ is the measured current and $\mathrm{V}_{\text {gap }}$ is the gap voltage. Both are measured for a same applied voltage lower than the breakdown voltage. The gap capacitance $\mathrm{C}_{\mathrm{g}}$ is then found to be equal to $1.8 \pm 0.2 \mathrm{pF}$.

In order to determine the equivalent capacitance of the wires, $\mathrm{C}_{\mathrm{w}}$, numerical simulations have been performed, using Simulink (MathWorks). The input voltage pulse is taken from measurements performed with a pulse of 500-ns duration and 4-kV magnitude. In this case, with a gap distance of 2-mm no discharge is observed.

From the simulations, $\mathrm{C}_{\mathrm{w}}$ is then determined by comparing the simulated voltage and current with the experimental ones, for each of the three wires. Figure 3 presents the best agreement obtained in the case of HRW-50mm for $\mathrm{C}_{\mathrm{w}}=1 \mathrm{pF}$.

TABLE I

SUMMARY OF THE EleCtRODES ElECTRICAL PROPERTIES

\begin{tabular}{lcc}
\hline \hline \multicolumn{1}{c}{ Electrode } & Resistance, $\mathrm{R}_{\mathrm{w}}(\mathrm{k} \Omega)$ & Capacitance, $\mathrm{C}_{\mathrm{w}}(\mathrm{pF})$ \\
\hline $\mathrm{CuW}$ & $1 \times 10^{-6}$ & 0 \\
$\mathrm{HRW}-50 \mathrm{~mm}$ & $12 \pm 2$ & $1 \pm 0.5$ \\
HRW-500mm & $120 \pm 20$ & $1 \pm 0.5$ \\
\hline \hline
\end{tabular}

Table I summarizes the equivalent resistance and capacitance of the three cables. Note that the capacitance of the HRWs are similar for both lengths. This unexpected result is probably due to the low capacitance of the material and the large uncertainty ( $1 \pm 0.5 \mathrm{pF}$ ). Consequently, the HRWs can be modeled as purely distributed resistors.

\section{B. Comparison between $H R W-50 m m$ and a $12 \mathrm{k} \Omega$ resistor}

In the case without discharge, the highly-resistive wires can be modeled as resistors. It is then interesting to compare the gap
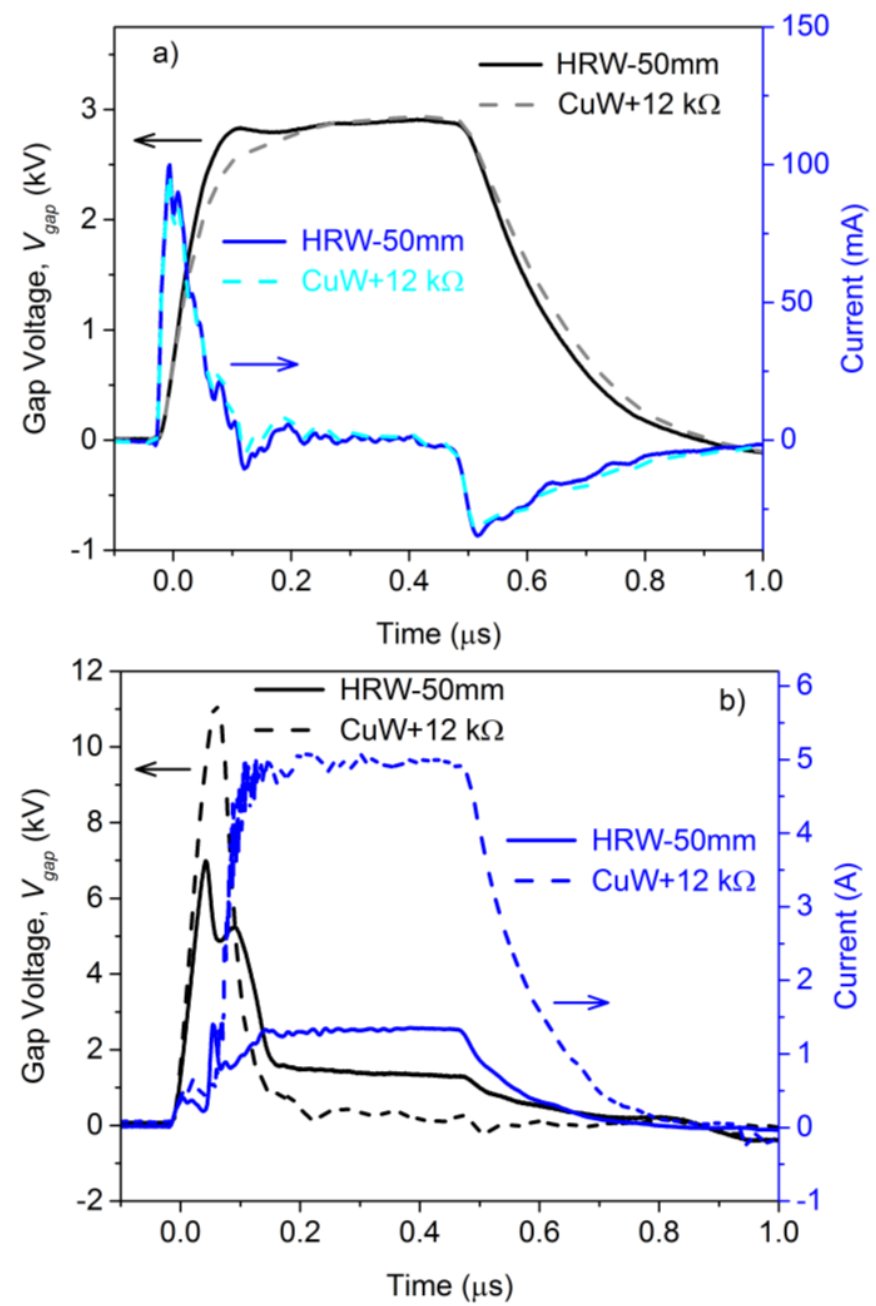

Fig. 4. Temporal evolution of the gap voltage and current for HRW-50mm and a copper wire with a $12 \mathrm{k} \Omega$ serial resistor, as the anode. For a) $4 \mathrm{kV}$ applied voltage (no discharge), and b) $14 \mathrm{kV}$ applied voltage (with discharge). 
voltage and current obtained for the HRW-50mm with a copper wire associated to a $12-\mathrm{k} \Omega$ resistor in serial (same equivalent resistance as the HRW-50mm).

Figure 4a presents the results obtained for an applied voltage of $4 \mathrm{kV}$, corresponding to a case without breakdown. The voltage and current waveforms are very similar for both electrodes. This confirms that the HRW-50mm can be modeled by a resistor of $12 \mathrm{k} \Omega$. However, for an applied voltage of 14 $\mathrm{kV}$, breakdown occurs with both electrodes. The gap voltage and current are then very different (see Fig. 4b). For the HRW$50 \mathrm{~mm}$ the current is about 5 times lower and the voltage $40 \%$ lower than those obtained for a copper wire associated to a resistor. Therefore, the distributed nature of the highly-resistive wires seems to strongly affect the electrical discharges. This confirms that RBDs cannot be considered as a simple additional resistor in the equivalent electrical circuit.

\section{Electrical characterization of the discharges obtained for pin anodes}

The electrical energy is determined from voltage and current measurements. First, the energy deposition in the gap by the discharge is determined from $V_{g a p}$ and the conduction current, $I_{c o n d}$, following the procedure detailed in [17]. From the total current, $I_{t o t}$, measured by the current probe, assuming that the current induced from electromagnetic interferences can be neglected (see [18]), the conduction current can be obtained by subtracting the displacement current, $I_{d i s}$ :

$$
I_{\text {cond }}=I_{\text {tot }}-I_{\text {dis }}
$$

For a given applied voltage, the displacement current is measured by increasing the gap distance up to $10 \mathrm{~mm}$, and therefore avoiding breakdown. Figure 5 shows an example of conduction current obtained for a total voltage of $10 \mathrm{kV}$. For the following results, only the conduction current is considered.

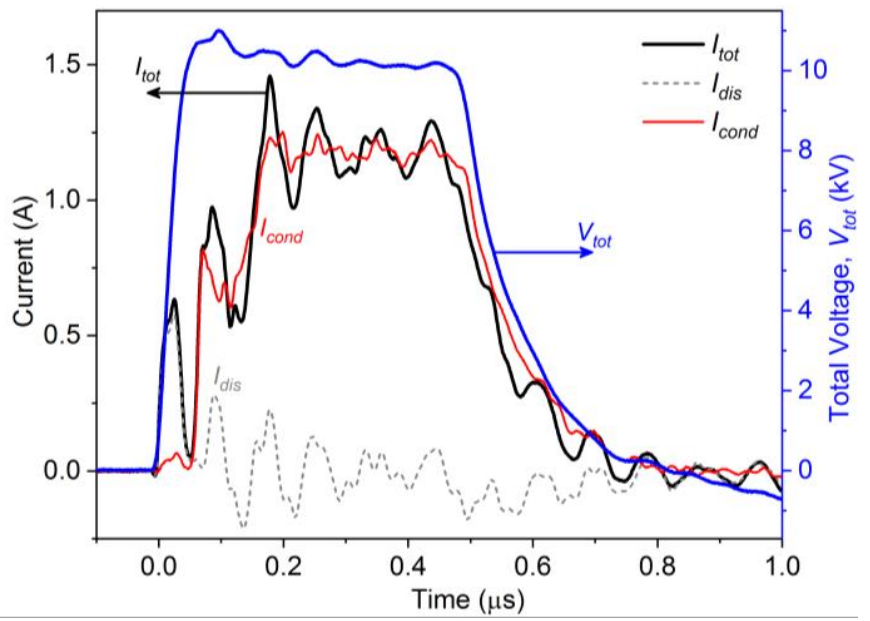

Fig. 5. Examples of total, displacement, and conduction currents as a function of time, along with the corresponding total voltage. The anode is the HRW$50 \mathrm{~mm}$.

After synchronization of the voltage and current waveforms, the energy deposition in the gap, $E_{g a p}$, is determined by integrating the product of the voltage and the conduction current over the discharge duration, $\tau$ :

$$
E_{\text {gap }}=\int_{0}^{\tau} V_{\text {gap }}(t) I_{\text {cond }}(t) d t
$$
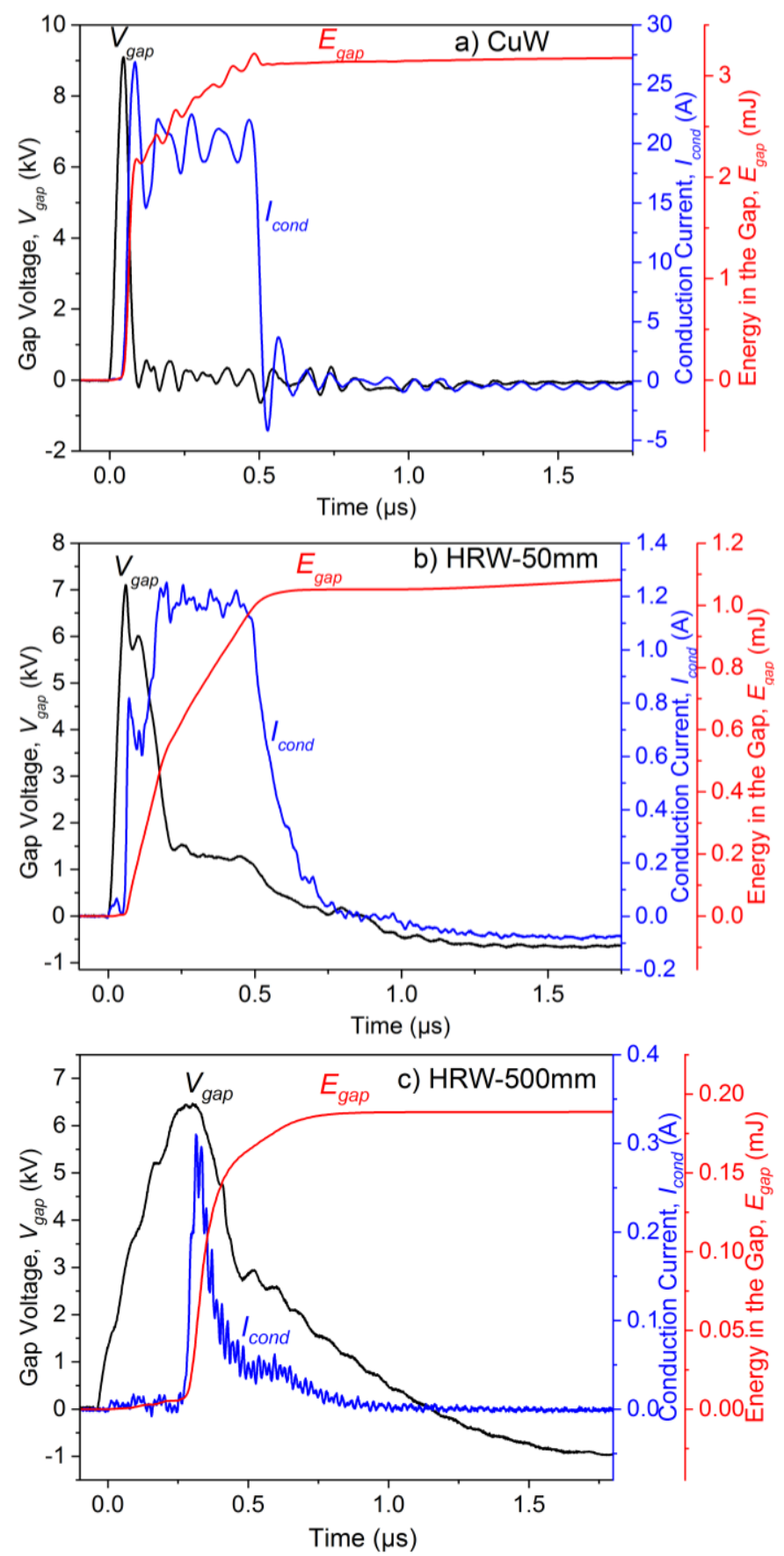

Fig. 6. Examples of the temporal evolution of $V_{\text {gap }}$ and $I_{\text {cond }}$, and the corresponding deposited energy in the gap; $E_{g a p}$, for the anode made of a) copper, b) $50 \mathrm{~mm}$ of highly-resistive wire, and c) $500 \mathrm{~mm}$ of highly-resistive wire. The applied voltage is $14 \mathrm{kV}$ and the gap distance is $2 \mathrm{~mm}$.

Examples of gap voltage, conduction current and the corresponding deposited energy in the gap obtained with $\mathrm{CuW}$, HRW-50mm and HRW-500mm as the anode, are presented in Figs. $6 \mathrm{a}, 6 \mathrm{~b}$ and $6 \mathrm{c}$, respectively. For an applied voltage of 14 $\mathrm{kV}$, the higher current intensity is obtained when using the $\mathrm{CuW}$ (Fig. 6a). The peak current reaches $25 \mathrm{~A}$, and then remains at about 20 A until the end of the pulse. The corresponding breakdown voltage is $9 \mathrm{kV}$ and the deposited energy in the gap is about $3 \mathrm{~mJ}$. When HRW-50mm is used as the anode, the breakdown occurs at a slightly lower voltage of $7 \mathrm{kV}$, then, $V_{g a p}$ decreases to a plateau of about $1 \mathrm{kV}$ (Fig. 6b). Compared to $\mathrm{CuW}, I_{\text {cond }}$ is significantly smaller, with an almost constant 
value of $1.2 \mathrm{~A}$ during the pulse. The corresponding $E_{\text {gap }}$ is about $1 \mathrm{~mJ}$, i.e., 3 times smaller than for $\mathrm{CuW}$. For the case with HRW-500mm (Fig. 6c), the breakdown occurs at about $6.5 \mathrm{kV}$, but the current is very different compared to $\mathrm{CuW}$ and HRW$50 \mathrm{~mm}$. Instead of a relatively constant value during the pulse, after a peak at $0.3 \mathrm{~A}$, it constantly decreases until the end of the pulse and no plateau is observed. Compared to what was obtained in [7], with a fluctuating current at $1 \mathrm{MHz}$, in this study, no pulsation of the discharge is observed. However, as there is no quantitative information regarding the electrical properties of the resistive barrier used in [7], it is difficult to further analyze this difference. The corresponding energy in the gap is about $0.2 \mathrm{~mJ}$, i.e., 15 times smaller than for the case with $\mathrm{CuW}$. This first result indicates that for a similar applied pulse, replacing a copper wire by a highly-resistive composite material wire could significantly reduce the energy of the electrical discharge, and therefore significantly decrease the ignition threat.

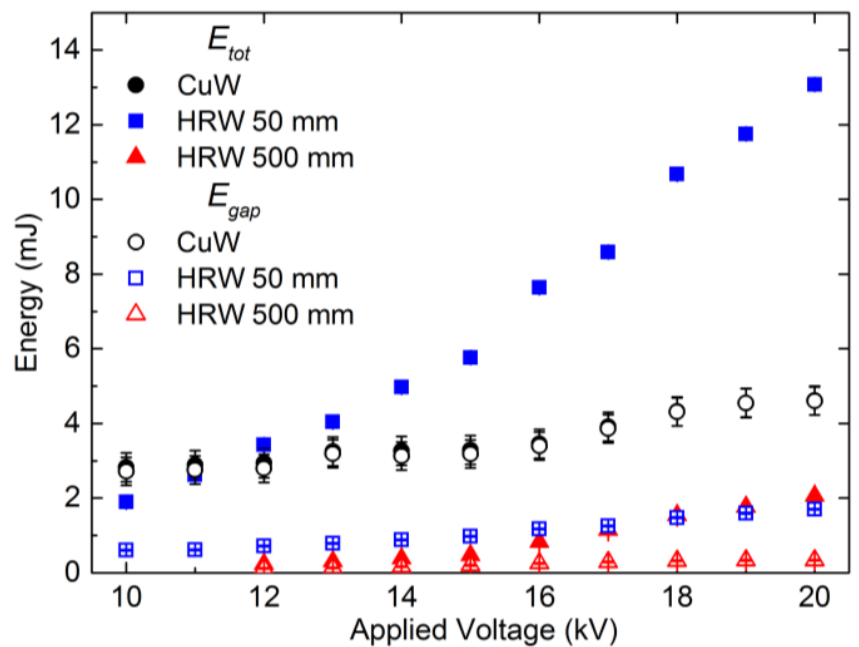

Fig. 7. Evolution of the total energy, $E_{\text {tot }}$, and the energy deposited into the gas, $E_{g a p}$, as a function of the applied voltage, for the three pin anodes. The gap distance is $2 \mathrm{~mm}$.

The electrical characterization of the discharges obtained for the three anodes has been carried out for the entire range of applied voltages that can be reached by the power supply, i.e. from 2 to $20 \mathrm{kV}$. In addition to the energy in the gap, $E_{g a p}$, another quantity of interest is the total energy deposited in both the tested wire and the gap. In the following sections, it is called the total energy, $E_{t o t}$, and it is determined by replacing $V_{g a p}$ by $V_{\text {tot }}$ in (4). Figure 7 summarizes the $E_{g a p}$ and $E_{t o t}$ obtained for the three anodes, with a 500-ns duration pulse and 2-mm gap distance. The error bars correspond to the standard deviation calculated from 10 different measurements.

For the three anodes, both the total energy and the energy in the gap increase linearly with the applied voltage. As expected, the values of the total and gap energies are very $\operatorname{similar}\left(E_{g a p} \approx\right.$ $\left.E_{t o t}\right)$ in the case of $\mathrm{CuW}$ (black circles), due to a negligible resistance of the wire. The energy in the gap varies from $2.9 \mathrm{~mJ}$ for an applied voltage of $10 \mathrm{kV}$ up to $4.5 \mathrm{~mJ}$, for an applied voltage of $20 \mathrm{kV}$. For the HRW-50mm cases (blue squares), the total energy is the highest, with up to $13 \mathrm{~mJ}$ for $20-\mathrm{kV}$ applied voltage. However, the maximal energy in the gap remains below $2 \mathrm{~mJ}$. Over the entire range of applied voltages investigated, only $15-25 \%$ of the total energy goes into the discharge. Compared to $\mathrm{CuW}$, in the cases of HRW-50mm, $E_{g a p}$ is about twice smaller. For the HRW-500mm cases (red triangles) both the total energy and the energy in the gap are smaller than for the two other anodes. The maximal total energy is about $2.1 \mathrm{~mJ}$, while the maximal energy in the gap is about $0.4 \mathrm{~mJ}$. Similarly than for the HRW-50mm cases, only $15-25 \%$ of the total energy goes into the discharge.
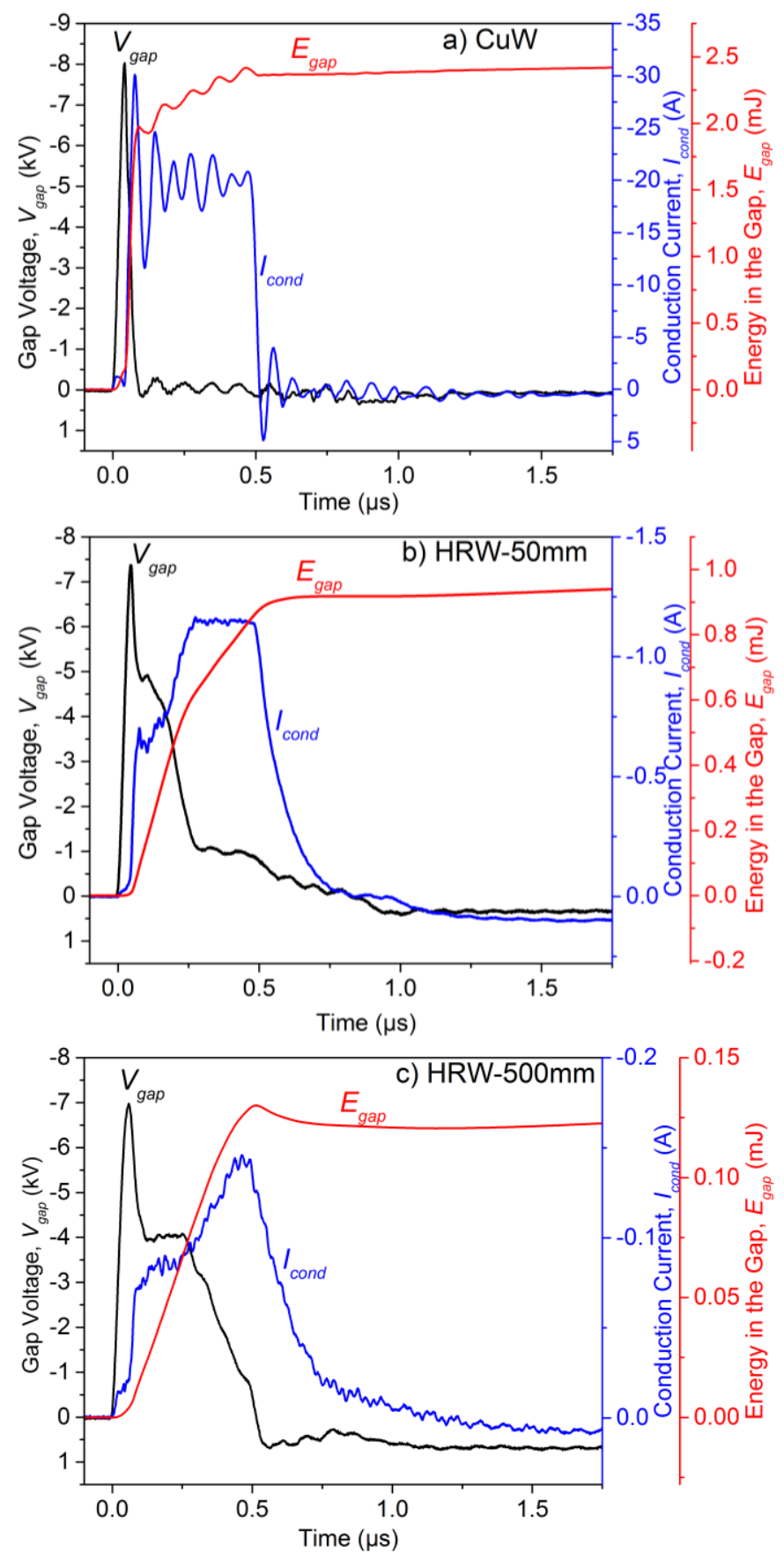

Fig. 8. Examples of the temporal evolution of $V_{\text {gap }}$ and $I_{\text {cond }}$, and the corresponding deposited energy in the gap; $E_{\text {gap }}$, for the cathode made of a) copper, b) $50 \mathrm{~mm}$ of highly-resistive wire, and c) $500 \mathrm{~mm}$ of highly-resistive wire. The applied voltage is $14 \mathrm{kV}$ and the gap distance is $2 \mathrm{~mm}$.

D. Electrical characterization of the discharges obtained for pin cathodes

By changing the polarity of the high-voltage pulse, it is possible to compare the effect of the cathode material on the 
discharge. In this case, the pin electrode is the cathode. Figure 8 presents examples of voltage and current waveforms, along with the corresponding energy deposited into the gap. Compared with the results obtained for the other polarity (when the pin is the anode), the electrical characteristics of the discharges are very similar. The current as well as the energy deposition in the gap are the highest in the case of the $\mathrm{CuW}$ cathode (Fig. 8a). They are the lowest in the case of the HRW$500 \mathrm{~mm}$ cathode (Fig. 8c). In the case of $\mathrm{CuW}$ and $\mathrm{HRW}-50 \mathrm{~mm}$, the first peak of current is followed by a plateau, typical of an arc. In the case of the HRW-500mm cathode, the maximal current is extremely low, about $0.15 \mathrm{~A}$, and it is observed at the end of the applied pulse.

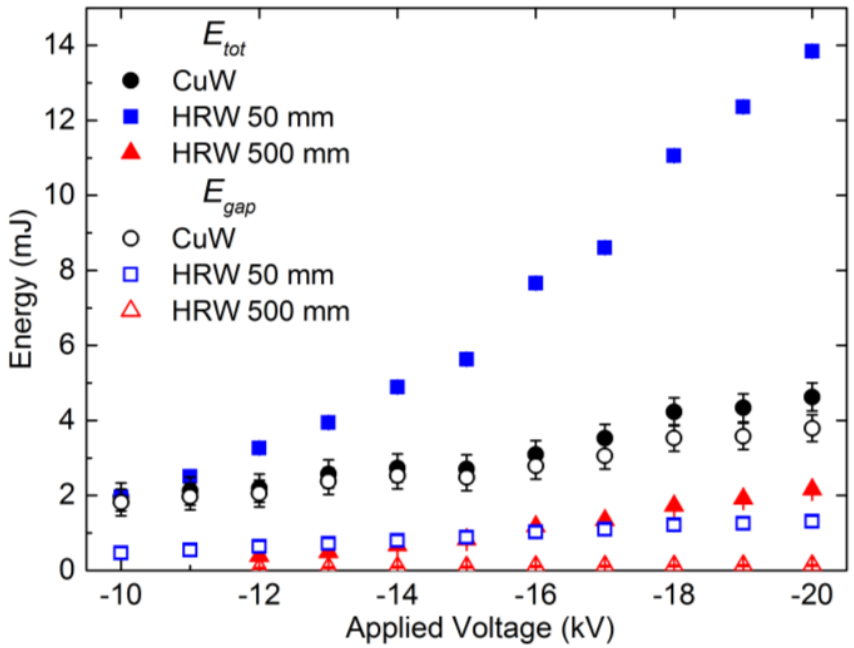

Fig. 9. Evolution of the total energy, $E_{\text {tot }}$, and the energy deposited into the gas, $E_{g a p}$, as a function of the applied voltage, for the three pin cathodes. The gap distance is $2 \mathrm{~mm}$.

Figure 9 summarizes the energy deposition in the gap, $E_{\text {gap }}$, and total, $E_{\text {tot }}$, for the three cathodes CuW, HRW-50mm and HRW-500mm, as a function of the applied voltage. The results are really close to what is obtained for the pin anodes. This similarity could be explained by the fact that the duration of the pulse is short, $500 \mathrm{~ns}$. Taking into account the asymmetry of the electrodes and the large value of the pressure multiplied by the gap distance (pd), a streamer-breakdown is probably the dominant mechanism of breakdown, for which the secondary emission from the cathode is insignificant [19].

These results for the various anodes and cathodes confirm that highly-resistive composite material can significantly reduce the energy of the electrical discharge, compared to metallic electrodes. For any polarity or length of the wire, the use of highly resistive material instead of copper reduces by more than a factor of three the energy deposition into the gas. This can be explained by energy dissipation by Joule effect in the RBD configuration. It is interesting to note that, the selfpulsing behavior that has been reported in previous studies [7] is not observed in the present study. Finally, regarding the discharge regimes, at the end of the 500-ns pulse, in the case of copper or short highly-resistive electrode, transition to an arc seems to have occurred, regardless of the polarity. In the case of long highly-resistive electrode, with a maximum current less than $500 \mathrm{~mA}$, the arc regime can be avoided.

\section{E. Visualization of the discharges}

In order to better characterize the effect of the electrode material on the discharges, time-resolved images of the propagation of the discharge during a pulse for the three anodes have been conducted. The intensified camera used in this study allows a minimal exposure time of $2 \mathrm{~ns}$, and a maximal repetition rate of $10 \mathrm{~Hz}$. In order to follow the temporal evolution of the breakdown and the subsequent electrical

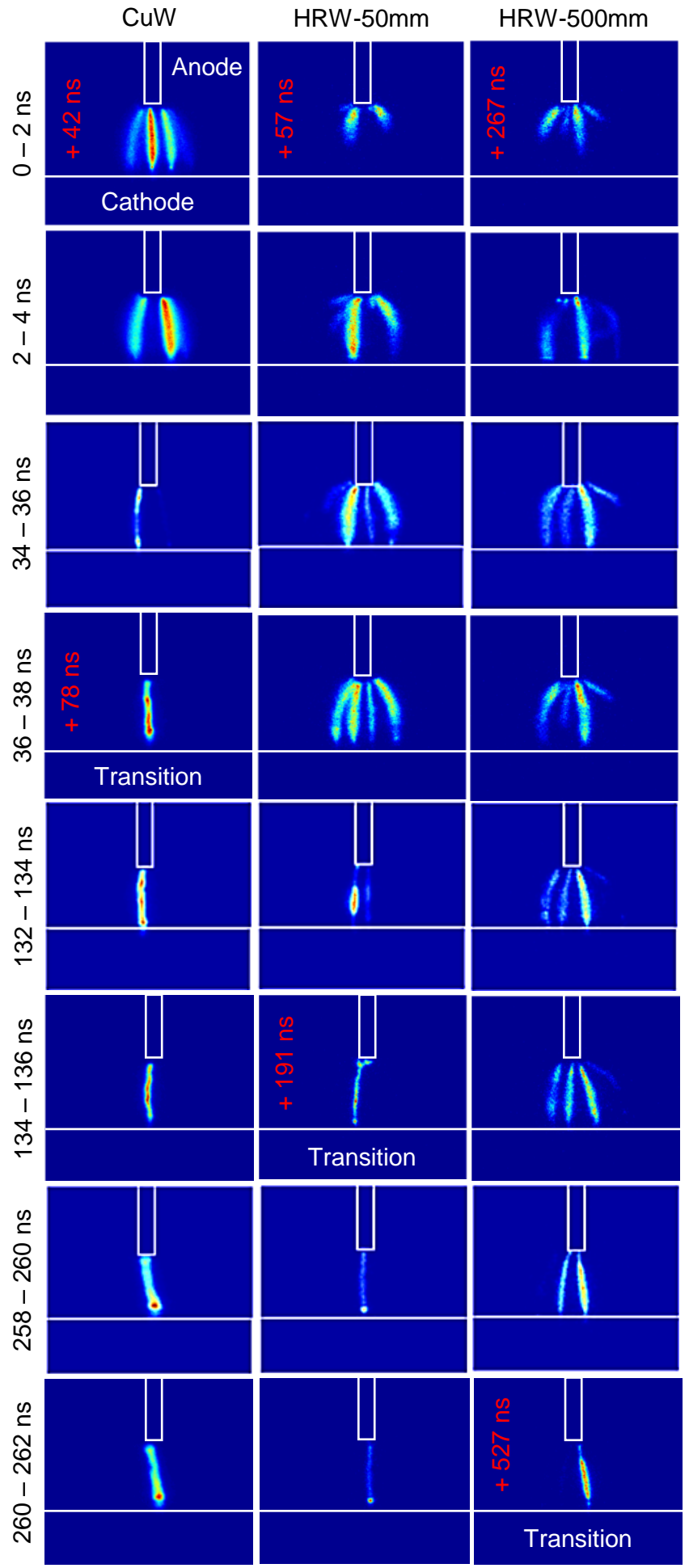

Fig. 10. Phase-locked images of the discharge propagation in the gap for the three anodes (14-kV applied voltage).The times in red correspond to the delays from the beginning of the voltage pulse. 
discharge, it is assumed that for a given configuration of electrodes and applied voltage, the discharges are reproducible. Therefore, synchronized with the high-voltage pulse, singleshot phase-locked images with an exposure time of $2 \mathrm{~ns}$ are recorded and presented as the temporal evolution of the discharges obtained for the given condition. Figure 10 presents examples of the temporal evolution of the discharges obtained for the three anodes CuW, HRW-50mm and HRW-500mm, for an applied voltage of $14 \mathrm{kV}$. This fixed applied voltage corresponds to a similar magnitude of the gap voltage (see Fig. 6 ), because the inter-electrode conditions (gas, pressure, temperature and electrode geometry) are kept fixed for all cases. In order to clearly see the shape of the discharges, the color map is ajusted for each image. Note that with this graphical representation, the light intensity from one image to another cannot be directly compared.

For the CuW (first column in Fig. 10), the breakdown is extremely fast. In $2 \mathrm{~ns}$, multiple filaments bridge the gap between the electrodes. This multi-filament phase last for $34 \mathrm{~ns}$, then a single filament is promoted, which lasts until the end of the applied pulse. For the HRW-50mm (second column in Fig. 10), the breakdown is slower. In the first $2 \mathrm{~ns}$, the filaments from the anode propagate until mid-gap only. In the second image (from 2 to $4 \mathrm{~ns}$ ), some of them reach the cathode. The same two phases (multi-filament followed by a single intense filament) than for the $\mathrm{CuW}$ can be observed. However, the transition multi-single filament occurs after $132 \mathrm{~ns}$. The breakdown obtained for the HRW-500mm (third column in Fig. 10), is similar to the one with HRW-50mm. However, the transition multi-to-single filament occurs at $260 \mathrm{~ns}$.

Compared to the reference case with the anode made of a copper braid, the propagation speed of the initial streamers in the case of RBDs is at least twice slower. This can be due to the differences in the rising time of the gap voltage for each electrodes (see for example Figs. 6a and 6c). At the beginning of a pulse, in the case of the RBDs the gap voltage is slightly smaller and its slope is lower than for the copper anode. As the geometry of the three cables is similar, the electric field close to the tip of the pin anode (where the breakdown process starts), is then also lower. As the propagation speed of a streamer increases with the electric field, the slower breakdown observed with RBDs, compared to the discharges with copper electrodes, seems induced by the voltage drop across the highly-resistive composite material.

For each electrode configuration, the transition multiplesingle filament corresponds to different delays from the beginning of the discharge. These delays, varying from $36 \mathrm{~ns}$ for the $\mathrm{CuW}$ up to $260 \mathrm{~ns}$ for the HRW-500mm does not correlate with any specific pattern of the current. However, compared to the beginning of the applied voltage pulse (in red on Fig. 10), these transitions correspond to the times at which the voltage drops below $3 \mathrm{kV}$ (see Fig. 6). Consequently, it seems that the transition multiple-single filament is driven by a decrease in the electric field.

\section{$F$. Correlation between the light emission and the electrical characteristics of the discharges}

The average light emission from the discharges has been determined from the phase-locked images. For the three wires, it has been compared with the instantaneous power and the conduction current of the corresponding discharge. Figures 11a, $11 \mathrm{~b}$ and $11 \mathrm{c}$ have been obtained for the CuW, the HRW-50mm, and the HRW-500mm, respectively. The green lines refer to the transition time from multiple to single filament phases. The light intensity is normalized with respect to the maximum intensity of the corresponding wire (different for each wire).
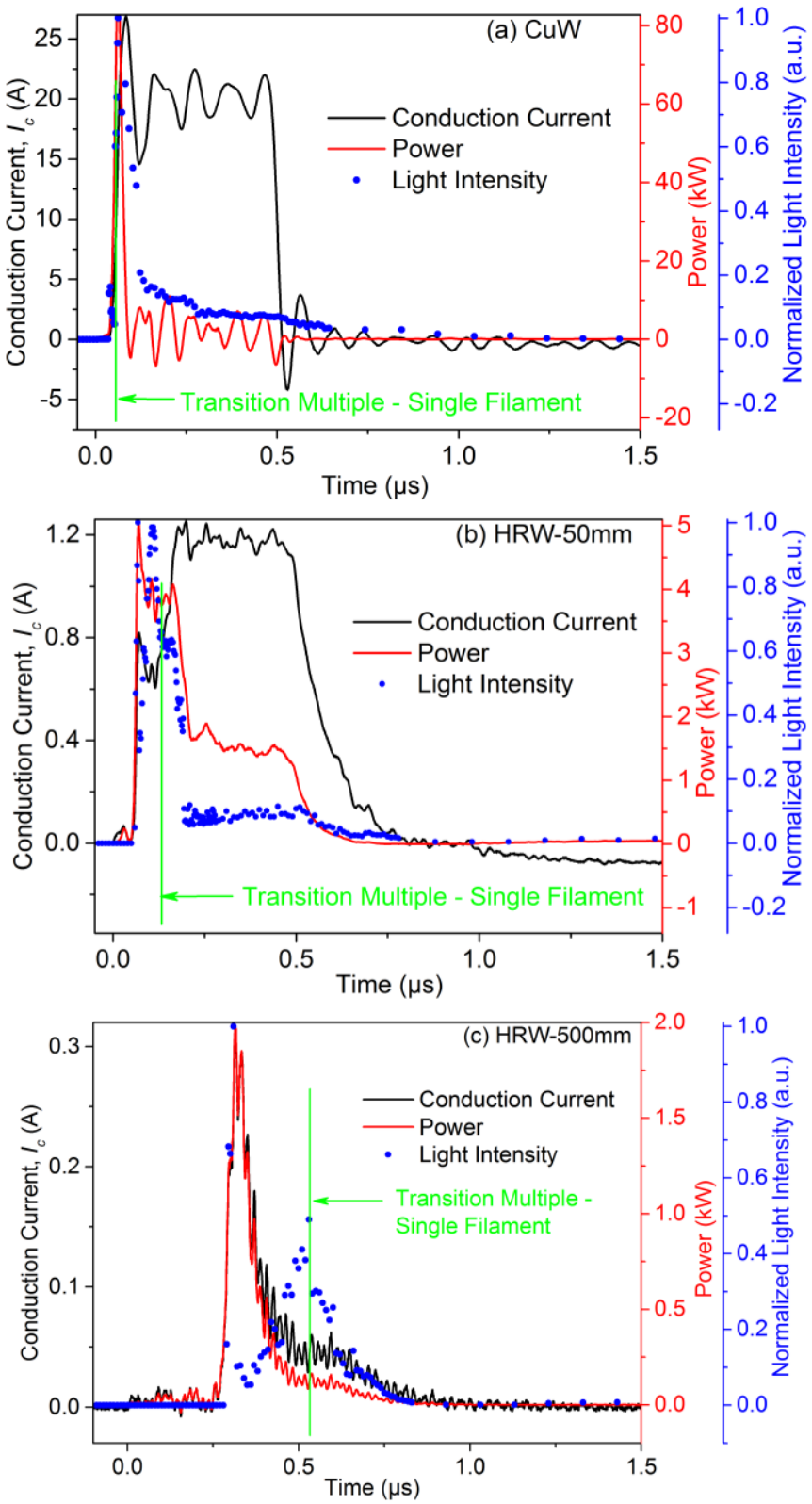

Fig. 11. Comparison between the temporal evolution of the conduction current, the instantaneous power and light intensities emitted by the discharge for an applied voltage of $14 \mathrm{kV}$, for (a) CuW, (b) HRW-50mm and (c) HRW$500 \mathrm{~mm}$.

Qualitatively, the temporal evolution of the light intensity from the discharge follows the temporal evolution of the instantaneous power. For the $\mathrm{CuW}$ and HRW-50mm (Figs. 11a 
and $11 \mathrm{~b}$, respectively) a first peak of light and instantaneous power is followed by a plateau of significantly lower magnitude. In the case of $\mathrm{CuW}$, the plateau of the instantaneous power is difficult to observe on the figure, due to the strong oscillations of the current measurements. The end of the initial peak of light intensity corresponds to the transition time from multi-filament to single filament discharges. A comparison of the light intensity of each filaments during the multi-filament regime, with the intensity of the single filament regime shows that the initial peak is not induced by more intense filaments but by a larger volume of discharges. The plateau, corresponding to the single filament regime, has the characteristics of the beginning of an arc, with an almost constant high current (about $20 \mathrm{~A}$ for $\mathrm{CuW}$, and $1.2 \mathrm{~A}$ for HRW-50mm). It lasts until the end of the applied pulse.

For the HRW-500mm (Fig. 11c), after the initial peak of light and instantaneous power, the shape of the light and instantaneous power diverges. However, during the measurements, it has been observed that the reproducibility between discharges with the HRW-500mm is not perfect. In this case, the phase-locked measurements are debatable and the comparison should not be pushed further. In any case, both the electrical measurements and the imaging of the discharges confirm that the use of HRW-500mm as an anode seems to be effective in avoiding the induction of an arc.

\section{CONCLUSION}

Electrical and optical characterization of the breakdown discharges produced by a 500-ns high-voltage pulse applied to copper and highly resistive electrodes have been performed. The discharges obtained in a pin-to-plane configuration are different if the electrodes are metallic or made of highlyresistive composite material (RBD configuration). The main differences are (i) the propagation speed of the initial streamers, (ii) the duration and magnitude of the initial current peak, as well as (iii) the delay between the discharge regimes obtained during the $500 \mathrm{~ns}$ of the pulse, and (iv) the energy deposition in the gas. These differences are not sensitive to the polarity and they can be explained by changes in the electrical circuit and by heat dissipation by joule effect. This information is valuable for safety designers as it shows that replacing metallic cables by highly resistive wires can reduce both the deposited energy and power density and might reduce the inadvertent ignition threat in flammable environments.

\section{REFERENCES}

W. C. Hall, "Electrostatic Dischargers for Aircraft," J. Appl. Phys., vol. 18 , no. 8, pp. 759-765, 1947.

K. Gligliotti, "Static electricity and aircraft," Wiley Encyclopedia of Composites, Second Edition. John Wiley \& Sons, Inc., 2012.

H. J. Gillis and P. B. Wilson, "Theoretical Analysis of Aircraft Electrostatic Discharge," USAAVLABS Technical Report 65-53.; U. S. Arm. Avi. Mat. Lab. Sci., Fort Eustis, Virginia, USA, 1965.

T. Kravchik, E. Sher, and J. B. Heywood, "From Spark Ignition to Flame Initiation," Combust. Sci. Technol., vol. 108, no. 1-3, pp. 130, 1995.

L. He, "Critical conditions for spherical flame initiation in mixtures with high Lewis numbers," Combust. Theory Model., vol. 4, no. 2, pp. 159-172, 2000.

[6] M. Laroussi, I. Alexeff, J. P. Richardson, and F. F. Dyer, "The

resistive barrier discharge," IEEE Trans. Plasma Sci., 2002.
I. Alexeff and M. Laroussi, "The uniform, steady-state atmospheric pressure de plasma," IEEE Trans. Plasma Sci., vol. 30, no. 1, pp. 174-175, 2002.

[8] K. D. Stephan, S. Ghimire, R. K. Smith, L. Komala-Noor, and N. Massey, "Transverse stabilization of atmospheric-pressure DC glow plasma in air with resistive barrier," IEEE Trans. Plasma Sci., vol. 39, no. 10, pp. 1919-1926, 2011.

[9] M. Thiyagarajan, A. Sarani, and X. Gonzales, "Atmospheric pressure resistive barrier air plasma jet induced bacterial inactivation in aqueous environment Atmospheric pressure resistive barrier air plasma jet induced bacterial inactivation in aqueous environment," $J$. Appl. Phys., vol. 113, no. 093302, 2013.

[10] H. S. Uhm, J. G. Kang, E. H. Choi, and G. S. Cho, "Sterilization of medical equipment and contaminated articles by making use of a resistive barrier discharge," J. Korean Phys. Soc., vol. 61, no. 4, pp. 551-556, 2012.

[11] X. Wang, Z. Li, J. Zeng, X. Zhang, and L. Lei, "Improvement of atmospheric water surface discharge with water resistive barrier," Plasma Chem. Plasma Process., vol. 33, no. 4, pp. 691-705, 2013.

[12] K. D. Stephan and G. Sheleg, "Directional propagation of resistivebarrier discharge above water," IEEE Trans. Plasma Sci., vol. 43, no. 2, pp. 501-505, 2015.

[13] A. Fridman, Plasma Physics and Engineering. New York, USA: CRC Press, 2004.

[14] H. Z. Fu, Y. J. Xie, and J. Zhang, "Analysis of corona discharge interference on antennas on composite airplanes," IEEE Trans. Electromagn. Compat., vol. 50, no. 4, pp. 822-827, 2008.

[15] J. F. Gieras, "Electrical Ignition of Fuel-Air Mixture in Aircraft Fuel Tanks Electrical Ignition of Fuel-Air Mixture in Aircraft Fuel Tanks," no. July 2013, pp. 17-24, 2017.

[16] M. Gherardi et al., "Practical and theoretical considerations on the use of ICCD imaging for the characterization of non-equilibrium plasmas," Plasma Sources Sci. Technol., vol. 24, no. 064004, 2015.

[17] D. L. Rusterholtz, D. A. Lacoste, G. D. Stancu, D. Z. Pai, and C. O. Laux, "Ultrafast heating and oxygen dissociation in atmospheric pressure air by nanosecond repetitively pulsed discharges," J. Phys. D. Appl. Phys., vol. 46, no. 46, 2013.

[18] D. Arnaud-Cormos, S. Kohler, D. Bessières, R.P. O'Connor, J. Paillol, P. Leveque, "Electrical measurements for nanosecond repetitively pulsed discharges", IEEE Trans. Plasma Sci., vol. 42, no. 7, 2014

[19] Raizer Y.P., "Spark and Corona Discharges," in Gas Discharge Physics, vol. 55, no. 10, Heidelberg, Germany: Springer, 1997, pp. 324-370.

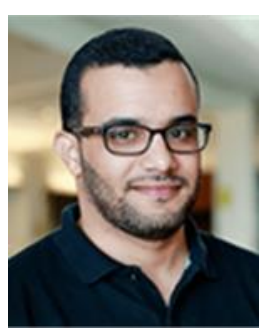

Abdeldjalil Reguig received the M.S. degree and the Ph.D. degree in 2012 and 2017 respectively, from the faculty of electrical engineering, University of Sidi Bel-Abbes, Algeria. Since 2017, he is postdoctoral fellow at the Clean Combustion Research Center, of the King Abdullah University of Science and Technology, in Saudi Arabia. His research mainly focus on plasma discharges at atmospheric pressure and applied electrostatics.

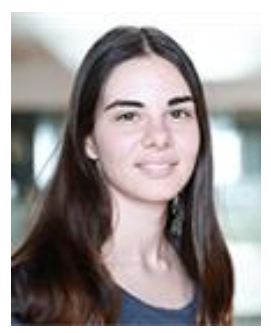

Belikse Ramljak received the B.S. degree in nuclear engineering from the City University of Hong Kong, in 2017. Since 2017 , she is a master student in mechanical engineering at the King Abdullah University of Science and Technology, in Saudi Arabia. Her research mainly focus on plasma discharges at atmospheric pressure. 


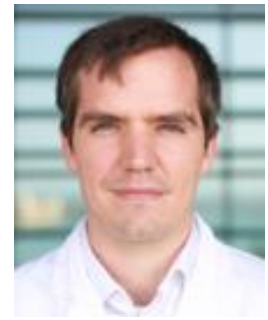

Karl Chatelain received the M.S. degree in energy and material sciences from the University of Orléans, France, in 2013 and the Ph.D. degree in chemical engineering from Université Paris-Saclay, in 2016. Since 2018, he is post-doctoral fellow at the Clean Combustion Research Center, of the King Abdullah University of Science and Technology, in Saudi Arabia. His research mainly focus on fundamental study of detonation.

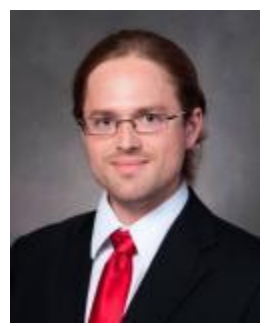

Jason Damazo graduated with a Ph.D. in Aeronautical Engineering from the California Institute of Technology where he focused on the interaction of gaseous detonations with structures. Since graduating, he has worked for The Boeing Company testing, modeling, and analyzing ignition, explosion, and other potentially high strain-rate events as they apply to aerospace product development and certification.

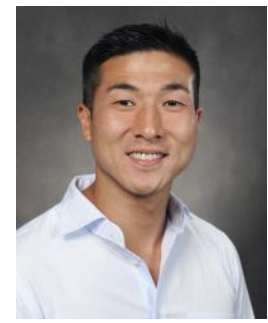

Eddie Kwon received the B.S. degree in physics from Oregon State University, USA, in 2004. Since 2005, he founded the Ignition Dynamics Laboratory at the Boeing Company. His research mainly focusses on determining the ignition threshold for novel ignition mechanisms to reduce accidental explosions in the aerospace industry.

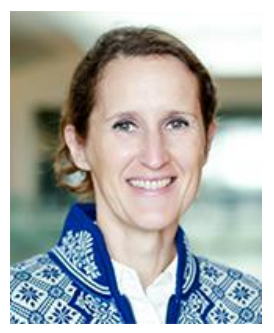

Deanna Lacoste received the M.S. degree in mechanical engineering from the University of Poitiers, France, in 1999 and the Ph.D. degree in mechanical engineering from the same university in 2002. Since 2016, she is an assistant professor of mechanical engineering at the Clean Combustion Research Center, of the King Abdullah University of Science and Technology, in Saudi Arabia. Her research mainly focus on plasma-assisted combustion, non-equilibrium plasma discharges at atmospheric pressure, control of flame dynamics and detonation. 